\title{
Letter
}

\section{Qualitative Analysis of Chaotic Behaviour in a Plasma System}

\author{
Ahmed Matouq Ahmed Hala \\ National Canter for Power Generation Technology, Energy \& Water Research Institute-KACST, Riyadh, Kingdom of Saudi Arabia \\ Email address: \\ ahala@kacst.edu.sa

\section{To cite this article:} \\ Ahmed Matouq Ahmed Hala. Qualitative Analysis of Chaotic Behaviour in a Plasma System. International Journal of Science and Qualitative \\ Analysis. Vol. 6, No. 2, 2020, pp. 16-18. doi: 10.11648/j.ijsqa.20200602.11
}

Received: May 13, 2020; Accepted: June 15, 2020; Published: July 4, 2020

\begin{abstract}
To analyze a physical plasma system, qualitative analysis methods should be applied first. Plasma systems components like the plasma source itself and its diagnostic tools must be studied to find out how these components interact to yield results describing the actual plasma system behaviour. For example, immersing a basic electric diagnostic instrument, such as the Langmuir probe, in a thermionically produced plasma source to measure plasma characteristic parameters constitutes a plasma system. When a time-sweep of the probe bias voltage is applied to the probe tip, plasma charge current is collected between the two probe bias voltage polarities. The resulting so-called I-V characteristics curve resembles the logistic curve proposed, previously, by Verhulst. The Verhulst logistic model curve described how population grow relative to available resources and formed the basis of modern chaos theory. In this letter, accounting for plasma charges population growth (or decay) as well as how they are sustained in a plasma system is discussed qualitatively. This is done without bearing additional assumptions as to the physical composition of the plasma charge itself. In addition, the findings here should modify the approach in interpreting Langmuir probe trace data that used before, only, an exponential fit to model the plasma charge current vs. probe bias voltage data. This allows for more fitting models to be implemented to analyze the behaviour of a variety of plasma systems.
\end{abstract}

Keywords: Physical Plasma, Charge, Langmuir Probe Trace, Verhulst Logistic Curve Model, Plasma Charge Current, Probe Bias Voltage, Energy, Chaos Theory

\section{Introduction}

Physical plasma is a quasineutral gas of charged and neutral particles that exhibits collective behaviour [1]. It is often referred to as the 4th state of matter. Plasma is ubiquitous in space and close to earth and can be studied using different models [2]. In addition, plasma sources and diagnostics were developed with increasing number of industrial and medical applications [3, 4].

The American scientist Irving Langmuir started his research in plasma physics in the early 20th century. He gave it its name after noticing, with his co-workers, its basic property as a medium where electromagnetic waves and particles are transported much like human body plasma which carries blood components [5]. Among his many contributions to plasma physics, Langmuir invented an electric probe to diagnose plasmas. Techniques of building and methods of interpreting the data of various types of this kind of electrical probes are given elsewhere [6].

On the other hand, and before Langmuir times, the Belgian mathematician Pierre-François Verhulst established his model to account for human population growth and its relationship to living resources by the middle of the 19th century [7]. Later, his model became the basis for the development of other logistic models that described various chaotic systems in other disciplines.

The American meteorologist Edward Lorenz computational efforts to forecasting weather helped in renewing interest in applying deterministic chaos theory in a variety of fields [8]. In plasma physics, microscopic chaotic features of plasma were studied as a part of describing complex aspects of plasma systems [9].

In this letter, chaotic behaviour of physical plasma is presented within the context of studying plasma systems that include two components. Namely; the plasma source and its diagnostic tools. A thermionically produced plasma source [10] and an electric probe, such as the Langmuir probe, are used as 
the main constructing components for this system.

The qualitative analysis method followed in this paper keeps physical assumptions to a minimum. In particular, and in regard to describing plasma charges, macroscopical description is given without positing additional physical explanation as to what a charge is beyond its attractive and repulsive nature.

\section{Inspection of Langmuir Probe Curves in Comparison with Verhulst Logistic Model}

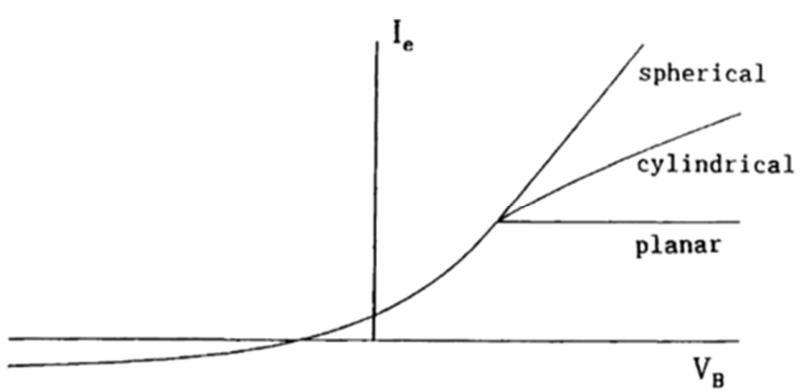

Figure 1. Typical I-V characteristics traces for various planar, cylindrical and spherical Langmuir probes [6].

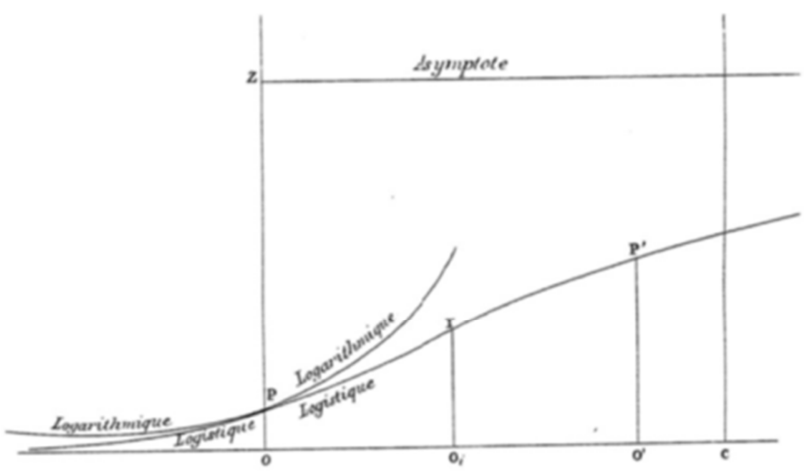

Figure 2. An original curve of Verhulst logistic model compared to a logarithmic scale curve [11].

Figure 1 shows typical Langmuir probe curves. Different probe trace shapes are due to each probe geometry and size which are optimized to collect sufficient plasma charge current vs. probe bias voltage.

Figure 2 represents the original Verhulst logistic curve that modeled population growth contrasted to the other contemporary exponential model.

When inspecting Langmuir probe curves in comparison with the Verhulst logistic model curve, the sudden and rapid increase in plasma current charge population, as the probe bias voltage approach the attraction polarity, indicates that the plasma system is behaving chaotically.

\section{Concept of Charge in Plasma}

The first modern mention of the existence of the concept of charge is due to Gilbert [12] in the context of explaining the difference between the physics of electric charge phenomenon and magnetic phenomenon. Coulomb defined the charge as a form of matter that experiences a force. No specific description (discrete or continuous) of charges was given. The description that prevailed, for a while, was of describing charges as a fluid with attractive and repulsive forces acting between them [13].

A general description of charges is given by defining the charge current as the rate of change of charges with time:

$$
I=\frac{d Q(t)}{d t}
$$

Where,

$I$ is the charge current.

$Q(t)$ is charge as a function of time.

$t$ is time.

This is a mathematical equation that does not impose any additional assumptions on the composition of charges, yet it is sufficient to account for the behaviour of flowing charges, or currents, in electrical systems. To date.

When interpreting Langmuir probe data, based on plasma system chaotic behaviour, the plasma attributes that exhibit its chaotic behaviour are its charge content value and how these charges are sustained. The Langmuir probe I-V trace measures the charges population rate of change vs. their sustenance energy as sensed by the probe bias voltage.

Further, the law of mass action can be utilized to arrive at the value of total charge value in a plasma system. This physicochemical law states that for all, but the most elementary chemical reactions, the rate of change of the concentration, or activity, of a reactant is directly proportional to the concentration, or activity, of this reactant [14].

Specifically,

$$
\frac{d Q}{d t}=-k Q(t)
$$

Where,

$k$ is the reaction rate.

When the Langmuir probe curve tends to saturate, as the time-sweep of probe bias voltage approaches the attractive polarity value, the plasma charge saturated current value represents the reaction equilibrium state. At this stage, the plasma system tends to stop producing additional charges.

Qualitative analysis of the charge concept in plasma leads to the identification of certain chaotic features in plasma systems. For example, an intuitive feature of these systems is that various plasma attributes co-exist in mixed states. Transitive mixed charge regions are clear in a Langmuir probe trace. Moreover, plasma charges are controlled by mixed types of forces such as Coulomb electric, magnetic, electromagnetic, pressure, hydrodynamic and magnetohydrodynamic forces.

\section{Mathematical Model}

The following mathematical model analyzes aspects of chaos theory related to the qualitative analysis of plasma systems following the Verhulst model. It assumes the following plasma system set-up: 
1. Thermionically produced large-scale plasma source.

2. Langmuir probe as a diagnostic tool.

The following is the dimensionless Verhulst logistic model equation [15]:

$$
\frac{Q(t)}{Q_{s a t}^{*}}=x(t)=\frac{1}{1+\left(\frac{1}{x_{0}}-1\right) e^{-r t}}
$$

$Q_{s a t}^{*}$ is the total saturation charge.

$x_{0}$ is the ratio of the charge value at the beginning of probe.

$r$ is the charge population growth parameter.

For different values of the parameter $r$, growth or decay curves in the plasma charge population are exhibited in Figure 3.

Where,
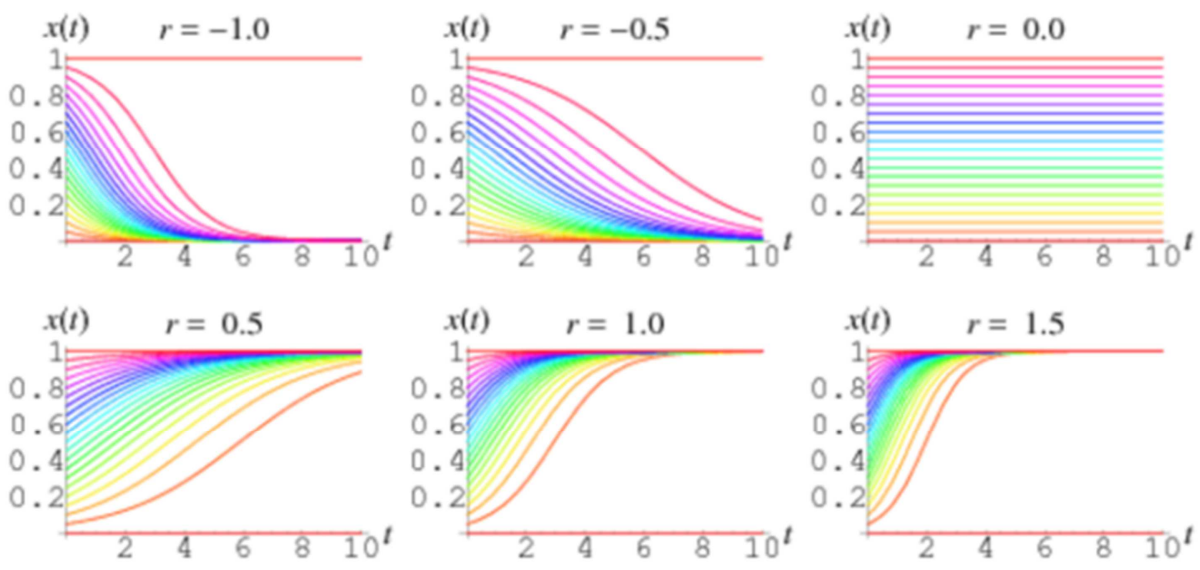

Figure 3. Curves of the logistic equation which can be used to model plasma charge population growth (or decay) rates as described by chaos theory [15].

\section{Concluding Remarks}

In a discipline that deals mainly with electrodynamics and electromagnetisms such as plasma physics, there is a need to further describe, in detail, aspects of plasma charge population and how it is produced. A qualitative analysis of a thermionically produced plasma source with a Langmuir probe as its diagnostic tool, has shown that plasma acts chaotically in such systems. Indeed, accounting for the plasma charge population rate of change and how it is sustained using elements of chaos theory helps in developing many useful applications for these plasma systems.

\section{Acknowledgements}

I would like to thank KACST administration for their continuous support in this work.

\section{References}

[1] Chen, Francis F. Introduction to plasma physics. Springer Science \& Business Media, (2012): 3.

[2] Alfven, Hannes. "Model of the plasma universe." IEEE transactions on plasma science 14, no. 6 (1986): 629-638.

[3] Lieberman, Michael A., and Alan J. Lichtenberg. Principles of plasma discharges and materials processing. John Wiley \& Sons, (2005).

[4] Chu, Paul K., and XinPei Lu, eds. Low temperature plasma technology: methods and applications. CRC Press, (2013).

[5] Fitzpatrick, Richard. Plasma physics: an introduction. CRC Press, (2014): 2.
[6] Hershkowitz, Noah. "How Langmuir probes work." Plasma diagnostics 1 (1989).

[7] Kint, Jos, Denis Constales, and André Vanderbauwhede. "Pierre-François Verhulst's final triumph." In The Logistic Map and the Route to Chaos. Springer, Berlin, Heidelberg, (2006): 13-28.

[8] Lorenz, Edward N. "Deterministic nonperiodic flow." In The Theory of Chaotic Attractors. Springer, New York, NY, (2004): 25-36.

[9] Escande, D. F. "Complexity and simplicity of plasmas." In AIP Conference Proceedings, vol. 1582, no. 1. American Institute of Physics, (2014): 22-34.

[10] Langmuir, Irving, and Chauncey Guy Suits. The Collected Works of Irving Langmuir: With Contributions in Memoriam, Including a Complete Bibliography of His Works - Volume 3. General Editor: CG Suits, Executive Editor: HE Way. Pergamon Press, 1961: 33.

[11] Verhulst, P. "La loi d'accroissement de la population." Nouv. Mem. Acad. Roy. Soc. Belle-lettr. Bruxelles Volume 18, no. 1 (1845): last page in the original copy of manuscript.

[12] Gilbert, William. De magnete. Courier Corporation, (1958).

[13] De Coulomb, Charles Augustin. "Cinquième Mémoire sur l'Electricité et le Magnétisme." Histoire de l'Académie Royale des Sciences (1787): 421-467.

[14] Guldberg, Cato Maximilian, and Peter Waage. Etudes sur les affinités chimiques. Brøgger \& Christie, 1 (1867).

[15] Weisstein, Eric W. "Logistic Equation.” Wolfram MathWorld. https://mathworld.wolfram.com/LogisticEquation.html (accessed May 5, 2020). 